\title{
Automation of clip localization in Digital Tomosynthesis for setup of breast cancer patients
}

\author{
Sook Kien $\mathrm{Ng}^{\mathrm{a}, \mathrm{b}, *}$, Yulia Lyatskaya ${ }^{\mathrm{a}}$, Dzmitry Stsepankou ${ }^{\mathrm{b}}$, Jurgen Hesser ${ }^{\mathrm{b}}$, \\ Jennifer R. Bellon ${ }^{a}$, Julia S. Wong ${ }^{a}$, Piotr Zygmanski ${ }^{a}$
}

\author{
a Department of Radiation Oncology, Brigham and Women's Hospital \& Dana Faber Cancer Institute, \\ Harvard Medical School, Boston, MA 02115, USA \\ ${ }^{\mathrm{b}}$ Department of Radiation Oncology, Mannheim Medical Centre, University of Heidelberg, Mannheim, Germany
}

Received 15 June 2011; received in revised form 30 November 2011; accepted 2 December 2011

Available online 28 December 2011

\section{KEYWORDS \\ Breast; \\ Patient setup; \\ Digital \\ Tomosynthesis; \\ CBCT}

\begin{abstract}
The objective of this study is to develop an automatic clip localization procedure for breast cancer patient setup based on Digital Tomosynthesis (DTS) and to characterize its performance with respect to the overall registration accuracy and robustness. The study was performed under an IRB-approved protocol for 12 breast cancer patients with surgical clips implanted around the tumor cavity. The registration of DTS images to planning CTs was performed using an automatic algorithm developed to overcome specific challenges of localization and registration of clips in the breast setup images. The automatic method consisted of autosegmentation (intensity-based thresholding with a priori knowledge about clip size and location to distinguish clips from bony features) and auto-registration of the segmented clip clusters. To determine the inherent accuracy and robustness of the registration algorithm, additional simulated DTS data was analyzed. The developed algorithm is efficient in removing false positives and negatives and provides an accuracy of better than $2.3 \mathrm{~mm}$ for $60^{\circ}$ and $3.3 \mathrm{~mm}$ for $40^{\circ}$ DTS. When incorporated in clinical software, this algorithm helps to facilitate fast and accurate setup evaluation with minimal dose delivered to patients.

(c) 2011 Associazione Italiana di Fisica Medica. Published by Elsevier Ltd. All rights reserved.
\end{abstract}

\footnotetext{
* Corresponding author. Department of Radiation Oncology, Brigham and Women's Hospital, Harvard Medical School, 75 Francis Street, ASBI-L2, Boston, MA 02115, USA.

E-mail address: sook_ng@dfci.harvard.edu (S.K. Ng).
}

\section{Introduction}

Patient setup with translational correction using linacmounted $\mathrm{kV}$ imaging systems can be achieved using orthogonal radiographs, Cone Beam Computed Tomography (CBCT) or Digital Tomosynthesis (DTS). Tomosynthesis is a new imaging technique that provides pseudo-three dimensional anatomy information of the patient while 
reducing the scan angles for on-board $3 \mathrm{D}$ imaging $[1,2]$. Unlike CBCT scans, tomosynthesis scans acquire projections over a much smaller scan angle $[1,3,4]$, typically between $10^{\circ}$ and $40^{\circ}$. This leads to a substantial reduction of imaging dose to the normal tissues, improved geometric clearance, and decrease of acquisition time compared to CBCT scans $[5,6]$. As a result of short scanning angles, a larger geometric clearance can be achieved with tomosynthesis. Although the feasibility of DTS and its advantages compared to orthogonal radiographs and CBCT for patient setup have been demonstrated $[1,3,6-10]$, DTS has not yet become a routine clinical technique. Commercial linac-mounted kV systems, developed for radiography or CBCT applications, generally do not offer readily available DTS acquisition, reconstruction, and registration modes of operation. Reconstructions carried out with the standard Feldkamp-Davis-Kress (FDK) Algorithm and shift-and-sum algorithms result in DTS images with pronounced artifacts which in turn may impede time-efficient and accurate 3D registration $[6,10]$. While new reconstruction algorithms such as Compressed Sensing CBCT may help to improve DTS image quality, they are still under development [11-13] and are not expected to be in clinical use until they become stable and time-efficient.

Due to the presence of reconstruction artifacts DTS images are uniquely different from and inferior to CBCT images. This difference may lead to a cumbersome and inefficient manual registration procedure, creating a major obstacle in routine clinical implementation of DTS imaging technique $[6,10]$. Automatic registration techniques based on mutual information [4] or correlation [9] are available but may not be adequate when registration of soft tissue or other subtle features is dominated by overlapping bony anatomy. Automatic registration of surgical clips in the setup of breast cancer patients is expected to be more challenging when the strong signals from the chest wall and couch interfere with the clip signal in the $\mathrm{kV}$ projections. To improve the robustness of automated registration methods, feature-based methods which utilize a priori knowledge about the feature shape, size, and intensity may be required. In the case of surgical clips, clip distortions in the DTS images are well understood [6] and therefore can be efficiently used for the purpose of image registration. An additional strategy which was used to navigate the registration of appropriate features in the reference images to the corresponding features in the treatment DTS images was to limit the search area to the volume defined by the maximum expected uncertainty of the laser-based setup. In most cases, it may be reasonable to limit the search area to the original locations of the clips plus $2.0 \mathrm{~cm}$ margin around the reference positions.

In this paper, we present a registration algorithm with the specific goal of being easily implementable inside the existing on-board imaging system and therefore it uses segmentation and correlation techniques that are straightforward to implement clinically.

\section{Materials and methods}

An automatic clip localization and registration algorithm was developed to facilitate DTS-based breast cancer patient setup, which utilizes a priori information characterizing surgical clip distortions in DTS images. The algorithm consists of two stages: (i) automatic segmentation of individual clips using intensity-based thresholding within a limited search region, and (ii) automatic correlation of clip clusters that efficiently accounts for false positives and negatives. Registration of the entire clip cluster rather than each clip individually is essential to increase the overall robustness of the registration algorithm, which allows for possible variations in the relative distances between the clips and the occurrence of false positives and negatives in the segmentation stage. The performance of the automatic DTS algorithm was evaluated using setup accuracy with respect to CBCT-based setup.

The clip signals in the DTS images are considerably elongated along the $\mathrm{kV}$ beam direction $[6,14]$ and characteristic dark areas are formed on their sides. However, the center of mass of the clip and the location of the highest intensity coincide with the physical clip center. The exact shape and size of the surgical clip (or rather clip artifact region) in the DTS images are unique for a given scan angle and the specific intensity level used for thresholding $[6,14]$. This information was pre-determined prior to the registration/segmentation of the clips based on planning CT. Furthermore, the locations of the clips in DTS images are expected to be within a given uncertainty margin relative to their locations in planning CT images. This margin is determined based on the knowledge of the uncertainty of laser-based setup and used as a search region in the clips segmentation. The automatic registration was performed by registering the clip cluster segmented in DTS images to the clip cluster segmented in the planning CT. The possibility of breast deformation and the occurrence of false positives and negatives are accounted for by distributing the reference clip locations with a Gaussian probability density. The parameters of the probability density depend on the magnitude of the uncertainties and are selected in a heuristic fashion.

\section{Patient data}

The study was performed under an Institutional Review Board approved protocol on 12 breast cancer patients. Each patient was implanted with $2-8$ surgical clips (HU in the range of 320-1450) in the vicinity of the tumor bed. Planning CTs were acquired per standard clinical protocol for all patients. Out of these 12 patients, only 5 patients had $C B C T$ scans during treatment. CBCTs were acquired using On-Board Imaging (OBI) system v.1.4 using a lowdose-thorax mode with $0.4 \mathrm{mAs}$ per projection, $110 \mathrm{kVp}$. For 7 patients that did not have CBCT scans (HU of clips implanted in 6 patients is listed in Table 1), the simulated data sets were generated. The simulated projections were generated using a ray casting algorithm in cone-beam geometry, without incorporating the effects of scattering, beam hardening, or changes of the photon fluence distribution due to a bow-tie filter. The projections were generated with $1^{\circ}$ incremental angle between projections, with the dimension of $1024 \times 768$ pixels and a pixel size of $0.39 \times 0.39 \mathrm{~mm}^{2}$. 
Table 1 Clip-based registration accuracy of $40^{\circ}$ DTS and $80^{\circ}$ DTS simulated images to planning CT. For one patient with clips with $\mathrm{HU} \leq 730$, the clips could not be segmented when using $40^{\circ}$ DTS images.

\begin{tabular}{|c|c|c|c|c|c|c|c|}
\hline \multirow[b]{2}{*}{$\underline{\text { Patient }}$} & \multirow[b]{2}{*}{ Clips' HU } & \multicolumn{3}{|c|}{ Registration using $40^{\circ}$ DTS } & \multicolumn{3}{|c|}{ Registration using $80^{\circ}$ DTS } \\
\hline & & $\Delta X_{\text {shift }}(\mathrm{mm})$ & $\Delta Y_{\text {shift }}(\mathrm{mm})$ & $\Delta Z_{\text {shift }}(\mathrm{mm})$ & $\Delta X_{\text {shift }}(\mathrm{mm})$ & $\Delta Y_{\text {shift }}(\mathrm{mm})$ & $\Delta Z_{\text {shift }}(\mathrm{mm})$ \\
\hline I & 850 & 0.3 & 1.3 & 1.3 & 0.3 & 1.3 & 1.3 \\
\hline II & 1430 & 1.3 & 1.2 & 2.5 & 1.0 & 0.1 & 2.5 \\
\hline III & 460 & \multicolumn{3}{|c|}{ Clips could not be segmented } & 0.3 & 1.3 & 1.3 \\
\hline IV & 730 & 0.3 & 2.6 & 1.4 & 0.0 & 2.6 & 1.3 \\
\hline V & 1230 & 1.1 & 1.3 & 0.1 & 1.3 & 0.1 & 0.1 \\
\hline VI & 1450 & 0.3 & 1.3 & 1.2 & 0.0 & 2.5 & 1.2 \\
\hline Average & & 0.6 & 1.5 & 1.3 & 0.5 & 1.3 & 1.3 \\
\hline $\operatorname{STD}(\sigma)$ & & 0.5 & 0.6 & 0.9 & 0.5 & 1.1 & 0.8 \\
\hline
\end{tabular}

\section{Study design}

To investigate the software performance alone without the confounding influence from imaging artifacts, clip migration, and breast tissue deformation, the algorithm was first applied on the simulated DTS data sets. In this simulation, CBCT projections were generated from the planning CT with known shifts of $5.0-15.0 \mathrm{~mm}$. The DTS images were reconstructed from these projections with the scan angles of $40^{\circ}$ and $80^{\circ}$. The reference images (original planning CTs) and DTS images were registered based on the segmented clips to determine the translational shift between the image sets. The difference between the obtained and applied shifts was used to evaluate the accuracy of the registration method.

In addition, DTS images were reconstructed from the CBCT projections of five patients as listed in Table 2. Since $\mathrm{CBCT}$ projection acquired from the $\mathrm{OBI}$ had higher resolution compared to the simulated projections generated from the planning CTs (pixel size: $1.25 \times 1.25 \mathrm{~mm}^{2}$; slice thickness: $2.5 \mathrm{~mm}$ ), the same clips were expected to have higher contrast in the DTS images reconstructed from the acquired CBCT projections. Therefore, smaller scan angles $\left(40^{\circ}\right.$ and $60^{\circ}$ ) were used instead of $80^{\circ}$ as in the case of the simulated data. Furthermore, surgical clips identified in the patients with CBCT images had slightly higher Hounsfield unit (HU) values $(\mathrm{HU} \geq 550)$ than those in the group of patients with only planning $C T$ images $(H U \geq 460)$. This provided an additional advantage for the CBCT-group of patients since clips with higher density (higher HU in full CBCT scan) are known to have higher contrast in the DTS images and therefore can be more easily and accurately segmented in the DTS images although it was previously demonstrated that the clips contrast is virtually independent of the scan angle [6]. The setup shifts determined from the registration between the DTS images and planning CT were compared to the shifts obtained from registration between CBCT and planning CT using clinical software, Offline Review 8.6. ${ }^{1} \mathrm{We}$ considered the shifts detected in the CBCT images as the baseline and used them to evaluate the accuracy of the shift detected in the DTS images.

\footnotetext{
${ }^{1}$ Varian Medical System, Inc.
}

\section{DTS image reconstructions}

DTS image reconstructions were performed using an in-house FDK-based algorithm implemented in the Compute Unified Device Architecture (CUDA) parallel programming environment. DTS imaging arc was tangential to the imaged breast (medial arc for the left breast; lateral arc for the right breast) while patients were in supine position. This setting is similar to the setting in our previous study [10]. In the current study, the center angle of the kV-source was: $\theta_{0}=315^{\circ}$ for the left breast cases and $\theta_{0}=225^{\circ}$ for right

Table 2 CM displacements of individual clips relative to the $\mathrm{CM}$ of the clips cluster in each direction were measured for each patient.

\begin{tabular}{|c|c|c|c|c|c|c|}
\hline & \multicolumn{3}{|c|}{$\begin{array}{l}\text { Registration using } 40^{\circ} \\
\text { DTS }\end{array}$} & \multicolumn{3}{|c|}{$\begin{array}{l}\text { Registration using } 60^{\circ} \\
\text { DTS }\end{array}$} \\
\hline & $x(\mathrm{~mm})$ & $y(m m)$ & $z(\mathrm{~mm})$ & $x(\mathrm{~mm})$ & $y(m m)$ & $z(\mathrm{~mm})$ \\
\hline \multirow[t]{2}{*}{ Patient A } & 1.2 & 2 & 0.9 & 1.7 & 1.5 & 1.1 \\
\hline & 1.9 & 1.3 & 0.7 & 2.0 & 1.2 & 0.8 \\
\hline \multirow[t]{5}{*}{ Patient B } & 1 & 0.2 & 0.9 & 1.6 & 0.1 & 0.1 \\
\hline & 0.5 & 0.8 & 1.4 & 0.1 & 2.3 & 0.1 \\
\hline & 0.2 & 2.6 & 0.1 & 0.1 & 0 & 0.5 \\
\hline & 0.1 & 0.3 & 0.8 & 0.5 & 0.3 & 1.4 \\
\hline & \multicolumn{3}{|c|}{$\begin{array}{l}\text { Clips could not be } \\
\text { segmented }\end{array}$} & 2.5 & 2.3 & 2.9 \\
\hline \multirow[t]{4}{*}{ Patient C } & 0.4 & 1.3 & 2.3 & 0.8 & 0.6 & 2.3 \\
\hline & 0.7 & 1.4 & 1.0 & 0.5 & 1.3 & 1.2 \\
\hline & 2.0 & 0.1 & 0.6 & 1.3 & 0.7 & 1 \\
\hline & 0.7 & 0.7 & 0.4 & 0.2 & 1.2 & 1.3 \\
\hline \multirow[t]{3}{*}{ Patient D } & 0.4 & 2.2 & 1.9 & 2.1 & 1.8 & 1.3 \\
\hline & 0.4 & 2.1 & 0.9 & 0.2 & 2.1 & 1.4 \\
\hline & 1.6 & 1.2 & 1.3 & 1.5 & 1.1 & 1.1 \\
\hline \multirow[t]{4}{*}{ Patient $\mathrm{E}$} & 1.6 & 0.1 & 1.2 & 1.1 & 0.5 & 1.2 \\
\hline & 1.1 & 0.9 & 1.5 & 1.6 & 1.1 & 1.4 \\
\hline & 1.8 & 0.3 & 2.7 & 2.4 & 0.3 & 2.9 \\
\hline & 0.9 & 0.5 & 0.3 & 1.3 & 0.8 & 1.8 \\
\hline Average & 1.0 & 1.1 & 1.1 & 1.2 & 1.1 & 1.3 \\
\hline STD & 0.6 & 0.8 & 0.7 & 0.8 & 0.7 & 0.8 \\
\hline
\end{tabular}


breast cases. The DTS image reconstructions were performed either directly from acquired CBCT projections or from 2D simulated projections calculated from the planning CT. Fig. 1 shows an example of a DTS image reconstructed from the CBCT projections of a breast cancer patient. The DTS coordinate system $(u, v, t)$ was set in reference to the DTS volume in which the DTS slices are oriented perpendicular to the beam direction when the gantry is at a specified center angle: $\theta_{0}=\left(\theta_{\text {start }}+\theta_{\text {stop }}\right) / 2$. The $t$-direction is along the imaging beam axis and corresponds to the DTS slice depth while the $(u, v)$ plane corresponds to the DTS beams-eye-view plane. The final reconstructed volume of the DTS images is $512 \times 512 \times 512$ voxels with a resolution of $1 \mathrm{~mm}^{3}$.

\section{Automatic clip localization}

Clip localization consists of two steps: clip segmentation followed by the registration of segmented clips. The segmentation and registration steps were performed on the $(u, v, t)$ coordinate frame because DTS images have high spatial resolution in the plane oriented perpendicular to the beam direction. The detected shift was later transformed into $(x, y, z)$ coordinate frame for comparison with the shift identified in Offline Review, where the $(x, y, z)$ coordinates correspond to the (left-right, anterior-posterior, cranial-caudal) direction.

\section{Segmentation}

In principle, surgical clips with HU much higher than that of soft tissue can be segmented using global thresholding approach. However, if a single-threshold method is used, in addition to clips but ribs and other structures with gray value higher than the defined threshold may be segmented as well. To overcome this problem several criteria were used to differentiate the clips from the ribs: (1) maximum search radius; (2) intensity-based threshold; and (3) rejection or acceptance of the thresholding results depending on the size of the segmented regions. Since patient setup errors greater than $2 \mathrm{~cm}$ are extremely rare in clinical setting, the search volume was limited to $2 \mathrm{~cm}$ beyond the original clip location in the reference images (Fig. 1a). This volume of interest was plotted in a histogram and the peak value of the histogram was identified. This value corresponds to the gray value of the highest occurrence, which is usually soft tissue in breast imaging.

To segment the clips with gray values higher than those of soft tissue, the threshold value was set to be $\sim 10 \%$ higher than the gray value of the detected peak. Finally, the size criterion was applied to the segmented region to exclude (1) large segmented structures ( $>20$ pixels), e.g. ribs or rib artifacts (Fig. 1b, f) and (2) small segmented structures ( $<5$ pixels) such as artifacts and calcifications (Fig. 1c, g). Clip regions occurring in multiple slices were correlated and ascribed to specific individual clips based on the connectivity of the next neighbor pixel in the slice direction. With the threshold values described above, segmented clip regions appeared in no more than 2-7 slices (slice spacing $=1.0 \mathrm{~mm}$ ) depending on their orientation with respect to the $t$-axis.

The center of mass of the segmented region was determined for each clip. Previous studies $[6,10]$ show that despite the clip's elongation along the beam direction ( $t$-axis) the center of mass (CM) is invariant (accurately corresponds to that determined in the reference image) a

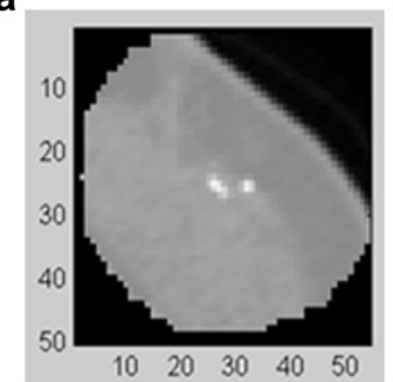

e

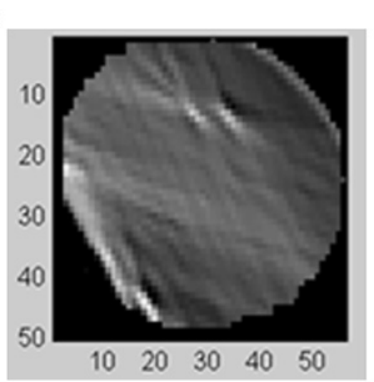

b

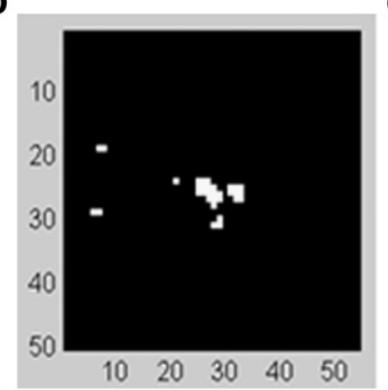

f

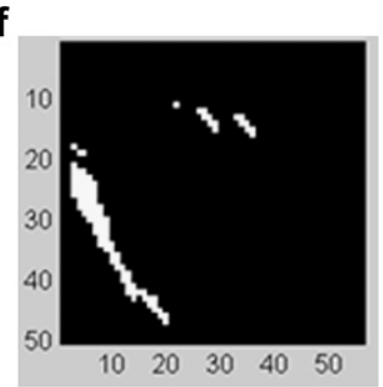

C

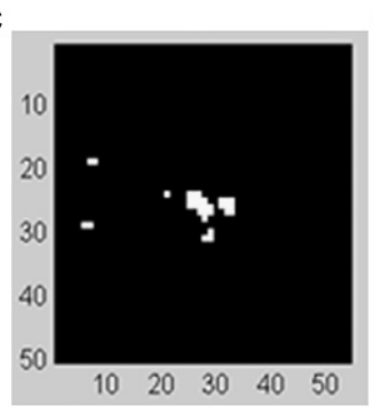

g

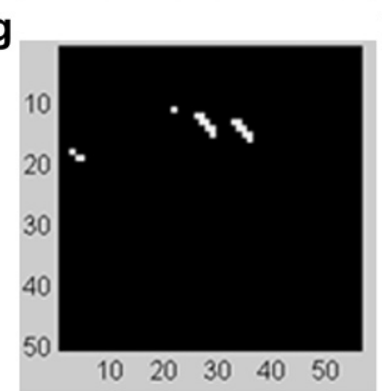

d

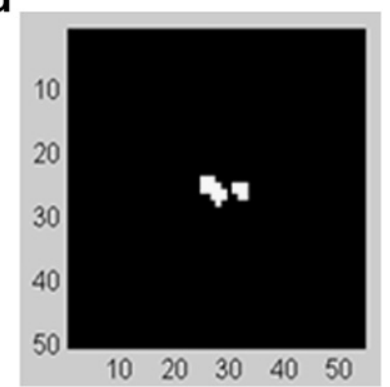

h

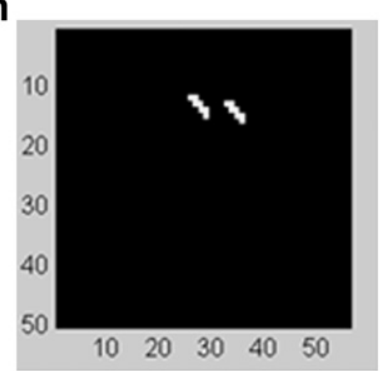

Figure 1 Illustration of the 4 steps in the automatic clip segmentation algorithm performed on a planning CT. (a-d) and $60^{\circ}$ DTS $(e-h)$ images. (a, e) The search region, limited to the clip location in the planning CT plus a $2 \mathrm{~cm}$ margin. (b, f) Results of the segmentation with applied threshold. Some artifacts were segmented in addition to the clips. (c, g) The large structures (ribs) were excluded after restricting the region size criterion of $<20$ pixels, (d, h) By requiring the region size to be $>5$ pixels, the small artifacts were excluded and only the clips were segmented. 
and the intensity of the clip is highest at its geometrical center. These observations were used to determine the relative shift between the two data sets during the image registration. The procedure described above was performed on both the treatment and reference images to localize the clip clusters in both data sets.

\section{Registration}

Occasionally there may be false positives and false negatives from the search algorithm (due to too many or too few segmented clips), producing unequal amounts of CM coordinates in the two data sets. This scenario was accounted for by registration of the entire cluster of clips rather than individual clips. Because of a finite intrinsic uncertainty in segmentation and the possibility of breast deformations, the registration is most effective if the CM of the clip is convolved with a Gaussian filter with a full width at half maximum(FWHM) characterizing the magnitude of those uncertainties. Accordingly, 3D Gaussian maps of the clip clusters with $0.2-0.4 \mathrm{~cm}$ standard deviation along each direction were formed for a final registration. The resulting location of the clip clusters in treatment DTS and reference CT images were then shifted with respect to each other. The shift which maximizes the overlap was used as the optimal setup shift.

\section{Verification of algorithm robustness}

The robustness of the algorithm was tested under conditions of false positives/negatives, possible tissue deformation that causes a change of the clips location relative to the center of mass of the whole clip cluster, and inherent uncertainties of the auto-segmentation algorithm. This is accomplished in two ways. First, we applied the algorithms on patient data to determine the translational shift and compared the detected shift to the baseline. The reproducibility of the determined shifts was used as the measure of robustness. Second, a simulation of registration errors was performed using clip locations found in planning CT with added known deformations. Specifically, patient specific clip locations were deformed by a known amount with respect to their reference values in the planning CT. Deformations were selected randomly with deformation amplitudes of $\pm(1-3) \mathrm{mm}$ according to the following equation:

deformed location = original location

\pm (deformation amplitude $\times$ random number $)$

where random number were generated in the range of $[-1,1]$. Therefore each of the clips' location was displaced by a different known amount (to account for potential tissue deformation) relative to the center of mass of the whole clips cluster. The two data sets were then registered, allowing the registration algorithm to determine the amount of shift due to the displacement applied.

\section{Results}

\section{DTS based on simulated projection data}

Table 1 shows the registration accuracy using $40^{\circ}$ and $80^{\circ}$ DTS images for six patients that were studied with the simulated projections. The HU of clips for these patients was $\geq 460$. In addition, one patient had clips of smaller size and with $\mathrm{HU}$ value of 320 , which could not be segmented in DTS images. The average registration accuracy using $40^{\circ}$ DTS images was $0.6 \mathrm{~mm} \quad(\sigma=0.5 \mathrm{~mm}), \quad 1.5 \mathrm{~mm}$ $(\sigma=0.6 \mathrm{~mm})$ and $1.3 \mathrm{~mm}(\sigma=0.9 \mathrm{~mm})$ along the $x-, y-$ and $z$-axis, respectively. Registration (segmentation) failed in one case when the clip HU was measured to be 460 . DTS images reconstructed using $80^{\circ}$ scan angle provide better results due to better image quality (better signal to noise ratio), especially in cases involving clips with low $\mathrm{HU}$ $(\mathrm{HU}=460)$. As shown in Table 1 , all six cases with clips $\mathrm{HU} \geq 460$ can be registered with the mean accuracy of $0.5 \mathrm{~mm}(\sigma=0.5 \mathrm{~mm}), 1.3 \mathrm{~mm}(\sigma=1.1 \mathrm{~mm})$, and $1.3 \mathrm{~mm}$ $(\sigma=1.1 \mathrm{~mm})$ along the $x-, y$ - and $z$-axis, respectively. When $40^{\circ}$ DTS images were used, clips with $\mathrm{HU}>700$ were reliably segmented, while clips with $\mathrm{HU} \geq 460$ were reliably segmented for DTS with scan angle $\geq 80^{\circ}$. The effective HU value of a clip depends on the material density and on its size (due to finite pixel size in CT and DTS images). According to a previous study on the clips used in breast patients such as AutoSuture Premium Surgiclip ${ }^{\text {TM }}$ S-9.0 and AutoSuture Premium Surgiclip ${ }^{\mathrm{TM}} \mathrm{M}-11.5$ with the lengths of $4.5 \mathrm{~mm}$ and $6.0 \mathrm{~mm}$ respectively have sufficiently large $\mathrm{HU}$ values ( HU600 and >HU1300 respectively) and can be reliably used for $40^{\circ}$ DTS imaging [6].

\section{DTS images based on acquired CBCT projections}

As mentioned above, the clips identified in the group of patients with acquired CBCT data had higher $\mathrm{HU}$ values than those identified in the group of patients with only planning CT (Table 2); thus minimizing the possibility of segmentation failure due to low contrast of the clips in DTS images for these patients. For patients with CBCT projections, reduced scan angles of $40^{\circ}-60^{\circ}$ were used for registration. Using the registration method described in the section Verification of Algorithm Robustness, CM displacements of individual clips relative to the $C M$ of the clips cluster were measured and are listed in Table 2. The maximal CM displacement of individual clips in registration of $60^{\circ}$ DTS images was $2.9 \mathrm{~mm}$ in all directions. When averaged over all patients, the values were: $1.2 \mathrm{~mm}(\sigma=0.8 \mathrm{~mm}), 1.1 \mathrm{~mm}(\sigma=0.7 \mathrm{~mm})$, and $1.3 \mathrm{~mm}(\sigma=0.8 \mathrm{~mm})$ along the $x-, y-$ and $z$-axis, respectively. Registration using $40^{\circ}$ DTS images showed that the maximal CM displacement of an individual clip for all patients was $2.2 \mathrm{~mm}$ in any direction. The averaged CM displacement of individual clips over five patients was $1.0 \mathrm{~mm}(\sigma=0.6 \mathrm{~mm}), 1.1 \mathrm{~mm}(\sigma=0.8 \mathrm{~mm})$, and $1.1 \mathrm{~mm}$ $(\sigma=0.7 \mathrm{~mm})$ along $x-, y$ - and $z$-axis, respectively.

Table 3 shows the results of registration using the CM of clips cluster. For registration using $60^{\circ}$ DTS images, the maximal shift deviation in any direction relative to the baseline was $2.3 \mathrm{~mm}$. The average value of the shift was $1.2 \mathrm{~mm}(\sigma=0.7 \mathrm{~mm}), 0.7 \mathrm{~mm}(\sigma=0.6 \mathrm{~mm})$ and $0.7 \mathrm{~mm}$ $(\sigma=0.3 \mathrm{~mm})$ along the $x-, y$ - and z-direction, respectively. When using $40^{\circ}$ DTS images the maximal shift deviation is $3.3 \mathrm{~mm}$ in any direction. The average value was $1.6 \mathrm{~mm}$ $(\sigma=1.4 \mathrm{~mm}), \quad 1.1 \mathrm{~mm} \quad(\sigma=0.4 \mathrm{~mm}) \quad$ and $\quad 0.8 \mathrm{~mm}$ $(\sigma=0.3 \mathrm{~mm})$ along the $x-, y$ - and $z$-direction, respectively. Table 4 shows the frequency of false positive and negative 
Table 3 Registration accuracy of $40^{\circ}$ DTS and $60^{\circ}$ DTS images based on registration of DTS images reconstructed from the acquired CBCT projections to planning CT images.

\begin{tabular}{|c|c|c|c|c|c|c|c|}
\hline \multirow[b]{2}{*}{ Patient } & \multirow[b]{2}{*}{ Clips' HU } & \multicolumn{3}{|c|}{ Registration with $40^{\circ}$ scan angle } & \multicolumn{3}{|c|}{ Registration with $60^{\circ}$ scan angle } \\
\hline & & $\Delta X_{\text {shift }}(\mathrm{mm})$ & $\Delta Y_{\text {shift }}(\mathrm{mm})$ & $\Delta Z_{\text {shift }}(\mathrm{mm})$ & $\Delta X_{\text {shift }}(\mathrm{mm})$ & $\Delta Y_{\text {shift }}(\mathrm{mm})$ & $\Delta Z_{\text {shift }}(\mathrm{mm})$ \\
\hline A & 550 & 1.2 & 1.6 & 0.2 & 1.5 & 0.9 & 1.0 \\
\hline B & 1350 & 3.3 & 1.2 & 0.5 & 2.3 & 1.7 & 0.3 \\
\hline C & 1380 & 2.7 & 1.0 & 0.8 & 0.5 & 0.2 & 0.7 \\
\hline D & 1350 & 0.2 & 1.1 & 0.9 & 0.8 & 0.6 & 0.9 \\
\hline $\mathrm{E}$ & 1380 & 0.8 & 0.5 & 2.2 & 0.8 & 0.1 & 0.6 \\
\hline Average & & 1.6 & 1.1 & 0.9 & 1.2 & 0.7 & 0.7 \\
\hline $\operatorname{STD}(\sigma)$ & & 1.4 & 0.4 & 0.8 & 0.7 & 0.6 & 0.3 \\
\hline
\end{tabular}

occurrences in registration of DTS images reconstructed from the acquired CBCT data. Only one false positive and two false negatives were observed in the acquired data. There were no false negatives or positives in three out of five cases. The occurrence of a false positive and negative did not result in any noticeable difference in the final shifts. In principle, one could expect a non-negligible error in shift when a false negative occurs in the case when only two clips are present in the patient. But in this study, the false negative scenario did not occur for the patient with only two clips (both clips were successfully segmented), therefore the registration was performed with small errors.

\section{Robustness of registration}

The accuracy of the automated registration software as presented in Tables 1-3, and its performance under the condition of false positives and negatives as listed in Table 4 , indicate that the registration algorithm is robust. The overall inherent uncertainty of the algorithm is about $1.0 \mathrm{~mm}$. It should be noted that the overall uncertainty of the DTS registration included the inherent uncertainty of CBCT vs planning CT registration (which defined the base(ine) and effects due to breast deformation, which might have occurred between the CT simulation and treatment days. Registration may fail in some cases when small features such as a wire placed on a patient's surface during the planning CT scan might appear within the ROI and is inadvertently segmented as a clip signal, as shown in Fig. $2(a-c)$. In this case, this wire appeared during image

Table 4 Frequency of false positive and negative occurrences in registration of DTS images generated from acquired CBCT projections.

\begin{tabular}{|c|c|c|c|c|c|}
\hline \multirow[b]{2}{*}{ Patient } & \multirow[b]{2}{*}{$\begin{array}{l}\text { No. } \\
\text { of clips }\end{array}$} & \multicolumn{2}{|l|}{$40^{\circ} \mathrm{DTS}$} & \multicolumn{2}{|l|}{$60^{\circ} \mathrm{DTS}$} \\
\hline & & $\begin{array}{l}\text { False } \\
\text { positive }\end{array}$ & $\begin{array}{l}\text { False } \\
\text { negative }\end{array}$ & $\begin{array}{l}\text { False } \\
\text { positive }\end{array}$ & $\begin{array}{l}\text { False } \\
\text { negative }\end{array}$ \\
\hline A & 2 & 0 & 0 & 0 & 0 \\
\hline B & 4 & 0 & 1 & 1 & 0 \\
\hline C & 4 & 0 & 0 & 0 & 0 \\
\hline D & 5 & 0 & 2 & 0 & 2 \\
\hline$E$ & 4 & 0 & 0 & 0 & 0 \\
\hline
\end{tabular}

registration as a false negative since the wire is not placed on a patient during treatment and therefore does not appear in the CBCT and DTS images. For this reason, it is recommended to manually remove the wire or similar signals from the planning CT data before image segmentation to avoid false positives during the registration steps. Simulation of deformations in the input data to the registration algorithm performed for the amplitudes $\pm(1-3 \mathrm{~mm})$ revealed uncertainties in the registration due to these conditions of about $1 \mathrm{~mm}$ in each direction.

\section{Discussion}

The presented methodology allows automatic registration of surgical clips in breast imaging using Digital Tomosynthesis images. The advantages of DTS include a lower dose to the patient, specifically to the contralateral breast and lung, faster acquisition time, and simpler image acquisition (avoidance of potential couch or patient collision with the gantry). However, without proper image processing, these advantages are not clinically realized due to significant distortions of clips and anatomy characteristic to DTS imaging. In our study, we were able to overcome these disadvantages by focusing on clips as fiducials for a breast cancer patient's setup and using a priori knowledge of clip's distortions in DTS. We observed that the clip's elongations (which occur along the beam axis direction) caused the clips' signal to overlap when clips were close to each other, impairing the registration effort. A larger number of well-distributed clips may improve the registration accuracy, but is not essential. The accuracy of automatic image registration evaluated for all patients in the study (including those with clips of lower HU values, overlapping clips, and images with false signals) was better than $3.3 \mathrm{~mm}$ for $40^{\circ}$ DTS and better than $2.3 \mathrm{~mm}$ for $60^{\circ}$ DTS with the average accuracy being better than $1.6 \mathrm{~mm}$ in any given direction. Thus, with surgical clips of sufficient $\mathrm{HU}$ value, this automatic method can be used for efficient patient setup verification in clinical setting to reduce the time and subjectivity based on DTS images of as low as $40^{\circ}$. For the clips with lower $\mathrm{HU}$ values, $60^{\circ}$ DTS images may be needed for some patients; this decision could be determined in the planning stage after acquisition of the planning CT. In addition, the selection of clips with higher $\mathrm{HU}$ values and their optimal placement during the surgical clip implantation procedure are clinically plausible, and would lead to 
a

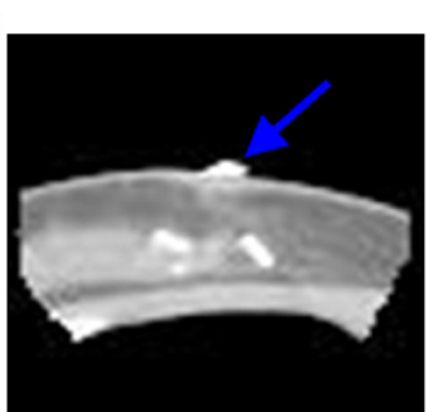

d

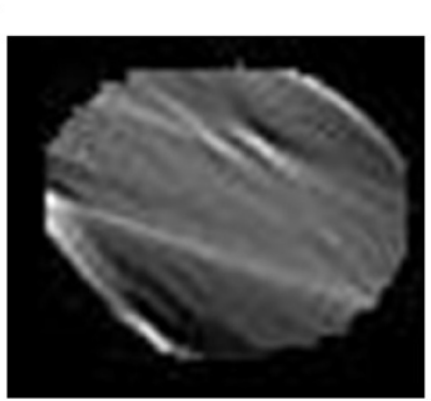

b

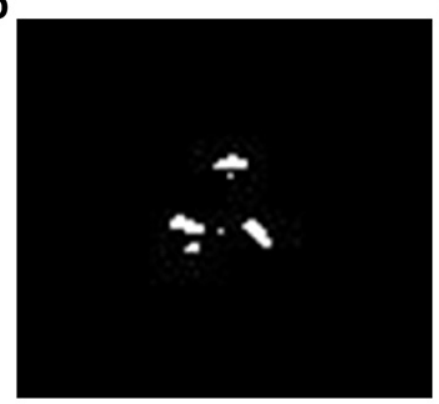

e

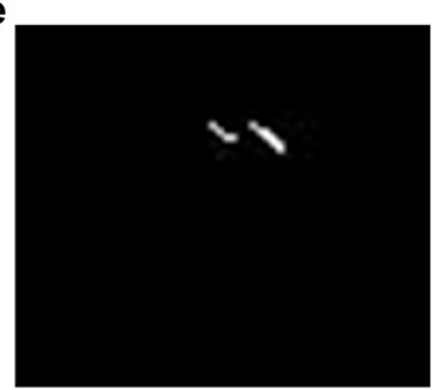

c

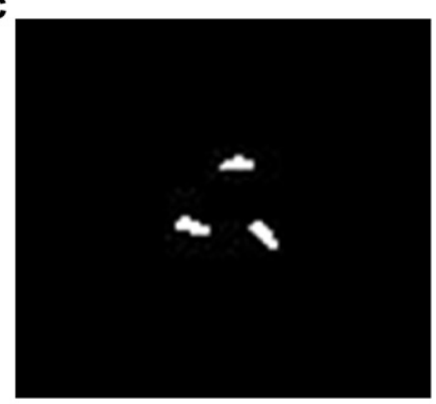

f

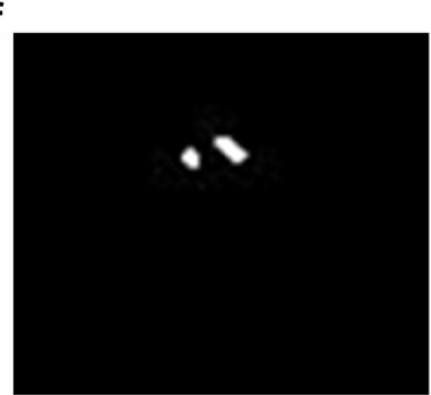

Figure 2 (a) Two surgical clips and a wire (indicated by the blue arrow) were present within the same range of gray value. (b, c) Both clips and wire were segmented. (d) Two clips appear elongated in the $40^{\circ}$ DTS images due to cross-plane leak of the signal. (e) During segmentation, more pixels were segmented in the DTS image due to structure elongation/deformation along the beam direction as compared to (f) pixels segmented from the planning CT image. (For interpretation of the references to color in this figure legend, the reader is referred to the web version of this article.)

fewer false negatives and positives and therefore improve the registration accuracy even further.

The final accuracy of clip-based auto-registration is limited by the accuracy of the auto-segmentation algorithm as presented in this study. This auto-segmentation is based on the gray-level thresholding with size restrictions for the search area. Our results show that this simple approach is satisfactory and would be straightforward to implement in currently available clinical treatment systems. However, other more elegant approaches, for instance based on $k$-nearest neighbor clustering techniques are plausible. The difficulty with such approaches is to find the suitable features in the feature space and create a large database for the training set in the clinical setting. With representative features and a large database, the clustering method is more robust; but for practical reasons, simple approaches are preferred.

\section{Conclusions}

An automatic method for surgical clips localization, segmentation, and registration in DTS images was developed and evaluated for breast cancer patient setup verification. The accuracy of the presented algorithm was tested for different DTS arc angles. The accuracy was $0.7-1.2 \mathrm{~mm}$ $(\sigma=0.3-0.7 \mathrm{~mm})$ in any direction using $60^{\circ}$ DTS images and $0.8-1.6 \mathrm{~mm}$ ( $\sigma=0.3-1.4 \mathrm{~mm})$ using $40^{\circ}$ DTS images. The developed algorithm is particularly effective in overcoming the disadvantages of DTS associated with clip signal distortions, and it is robust against false positive and false negative signals. The presented simple clip-based registration technique is feasible for automatic registration that minimizes user subjectivity as opposed to a manual registration method and thus improves efficiency of patient setup procedure. When incorporated in clinical software, this algorithm may help facilitate fast and accurate setup evaluation with minimal dose delivered to the patients. Thus its significance must be judged not only by the overall accuracy of the setup but also by its efficiency.

\section{Acknowledgments}

The authors would like to acknowledge the Kaye's Scholars Grant for the support of this research project.

\section{References}

[1] Godfrey DJ, Yin FF, Oldham M, Yoo S, Willett C. Digital tomosynthesis with an on-board kilovoltage imaging device. Int J Radiat Oncol Biol Phys 2006;65:8-15.

[2] Dobbins 3rd JT, Godfrey DJ. Digital X-ray tomosynthesis: current state of the art and clinical potential. Phys Med Biol 2003;48:R65-106.

[3] Yoo S, Wu QJ, Godfrey D, Yan H, Ren L, Das S, et al. Clinical evaluation of positioning verification using digital tomosynthesis and bony anatomy and soft tissues for prostate imageguided radiotherapy. Int J Radiat Oncol Biol Phys 2009;73: 296-305.

[4] Godfrey DJ, Ren L, Yan H, Wu Q, Yoo S, Oldham M, et al. Evaluation of three types of reference image data for external beam radiotherapy target localization using digital tomosynthesis (DTS). Med Phys 2007;34:3374-84.

[5] Winey B, Zygmanski P, Lyatskaya Y. Evaluation of radiation dose delivered by cone beam CT and tomosynthesis employed 
for setup of external breast irradiation. Med Phys 2009;36: 164-73.

[6] Buehler A, Ng SK, Lyatskaya Y, Stsepankou D, Hesser J, Zygmanski P. Evaluation of clip localization for different kilovoltage imaging modalities as applied to partial breast irradiation setup. Med Phys 2009;36:821-34.

[7] Zhang J, Wu QJ, Godfrey DJ, Fatunase T, Marks LB, Yin FF. Comparing digital tomosynthesis to cone-beam CT for position verification in patients undergoing partial breast irradiation. Int J Radiat Oncol Biol Phys 2009;73:952-7.

[8] Wu QJ, Godfrey DJ, Wang Z, Zhang J, Zhou S, Yoo S, et al. Onboard patient positioning for head-and-neck IMRT: comparing digital tomosynthesis to kilovoltage radiography and conebeam computed tomography. Int J Radiat Oncol Biol Phys 2007;69:598-606.

[9] Winey BA, Zygmanski P, Cormack RA, Lyatskaya Y. Balancing dose and image registration accuracy for cone beam tomosynthesis (CBTS) for breast patient setup. Med Phys 2010;37: 4414-23.
[10] Lyatskaya Y, Buehler A, Ng SK, Wong J, Bellon JR, Zygmanski P. Optimal gantry angles and field sizes in kilovoltage cone-beam tomosynthesis for set-up of women with breast cancer undergoing radiotherapy treatment. Radiother Oncol 2009;93: 633-8.

[11] LaRoque SJ, Sidky EY, Pan X. Accurate image reconstruction from few-view and limited-angle data in diffraction tomography. J Opt Soc Am A Opt Image Sci Vis 2008;25:1772-82.

[12] Nett B, Tang J, Leng $S$, Chen GH. Tomosynthesis via total variation minimization reconstruction and prior image constrained compressed sensing (PICCS) on a C-arm system. Proc Soc Photo Opt Instrum Eng 2008;6913. nihpa92672.

[13] Pan X, Sidky EY, Vannier M. Why do commercial CT scanners still employ traditional, filtered back-projection for image reconstruction? Inverse Probl 2009;25:1230009.

[14] $\mathrm{Hu} \mathrm{YH,} \mathrm{Zhao} \mathrm{B,} \mathrm{Zhao} \mathrm{W.} \mathrm{Image} \mathrm{artifacts} \mathrm{in} \mathrm{digital} \mathrm{breast}$ tomosynthesis: investigation of the effects of system geometry and reconstruction parameters using a linear system approach. Med Phys 2008;35:5242-52. 